\title{
Características y desarrollo del acogimiento familiar en dos países con fuerte tradición de acogimiento residencial: España y Portugal*
}

Characteristics and Development of Foster Care in Two Countries with a Strong Tradition of Residential Care: Spain and Portugal

Recibido: enero 8 de 2013 | Revisado: junio 5 de 2013 | Aceptado: febrero 13 de 2014

\author{
MÓNICA LÓPEZ** \\ University of Groningen, The Netherlands \\ Paulo Delgado *** \\ Instituto Politécnico do Porto, Portugal \\ JoÃo M. S. CARVALHO ***** \\ Instituto Universitário da Maia (CICS.NOVA), Portugal \\ JORGE F. DEL VALLE****** \\ Universidad de Oviedo, España
}

RES U MEN

Los procesos de acogimiento familiar que se producen en cada país dependen de factores históricos y culturales que dan lugar a grandes diferencias internacionales. A pesar de que las comparativas internacionales ofrecen un medio de intercambio de experiencias, lo que permite el aprendizaje mutuo y la transferencia de buenas prácticas, a menudo encontramos enormes barreras para su realización debido, entre otros factores, al limitado acceso a los datos sobre la práctica de la protección infantil. Frente a estas limitaciones,

doi:10.11144/Javeriana.UPSY13-3.cdaf

Para citar este artículo: López, M., Delgado, P., Carvalho, J. M. S. \& Del Valle, J. F. (2014). Características y desarrollo del acogimiento familiar en dos países con fuerte tradición de acogimiento residencial: España y Portugal. Universitas Psychologica, 13(3), 865-880. http://dx.doi.org/10.11144/ Javeriana.UPSY13-3.cdaf

* Agradecimientos: Los autores desean expresar su agradecimiento al Ministerio de Trabajo y Asuntos Sociales de España por el financiamiento del proyecto CN-06-142 y al InED - Centro de Investigação e Inovação em Educação do Instituto Politécnico do Porto, Portugal, por el apoyo económico recibido.

*** Correspondencia: University of Groningen, Faculty of Behavioral and Social Sciences, Department of Special Education and Youth Care. Groote Rozenstraat 38, NL-9712 TJ Groningen, The Netherlands.Correo electrónico: m.lopez.lopez@rug.nl

**** Instituto Politécnico do Porto, Portugal. Escuela Superior de Educación. Correo electrónico: pdelgado@ese.ipp.pt

******* Instituto Universitário da Maia (UNICES), Portugal. Correo electrónico: jcarvalho@ismai.pt

${ }^{* * * * * * *}$ Universidad de Oviedo, España. Facultad de Psicología. Correo electrónico: jvalle@uniovi.es este artículo ofrece una investigación comparativa realizada en España y Portugal, cuyo objetivo ha sido la evaluación del acogimiento familiar en dos países donde la investigación en el ámbito de la protección infantil ha sido tradicionalmente desatendida. Sobre una muestra de 357 casos en España y 289 en Portugal, se realizó un estudio del perfil de características de los niños acogidos, las familias de origen y los acogedores, así como de los procesos de acogida en ambos países. La comparativa reveló importantes diferencias relacionadas con la mayor edad al inicio del acogimiento de los niños en España; el perfil de los acogedores, con edades más avanzadas, un bajo nivel educativo y llevando a cabo acogidas de más de un niño simultáneamente en Portugal. El estudio comparado nos ha permitido identificar áreas que requieren mayor atención en ambos países, como la renovación generacional del banco de acogedores en Portugal o la necesidad de acelerar los procesos de toma de decisiones para la entrada en acogimiento familiar en España. Se pretende que este artículo sirva de aliento para la recogida de datos y comparación con otros países iberoamericanos.

Palabras clave

protección infantil; investigación comparativa; acogimiento familiar; medidas de separación familiar

\section{A B S T R A C T}

Fostering processes that occur in each country depend on historical and cultural factors that give rise to large international differences. Despite the fact that international comparisons offer a way of exchanging experiences, allowing mutual learning and transfer of good practice, we often encounter major barriers for its completion due to limited access to data on child protection practice, among other factors. Given these limitations, this article provides a comparative study conducted in Spain and Portugal. The aim 
is the evaluation of family foster care interventions in two countries where research in the field of child protection has traditionally been neglected. The profile of foster children, families of origin and foster families, as well as the features of the fostering processes were studied in a sample of 357 cases in Spain and 289 in Portugal. The comparison revealed significant differences related to the older age of Spanish foster children at the beginning of the foster placement. The profile of foster carers revealed older age, low educational level and frequent situations of multiple placements in Portugal. The comparative study has identified areas that require further attention in both countries, such as the generational shift of foster carers in Portugal or the need to speed up the decisionmaking processes for entry into a family foster placement in Spain. This article intends to be an encouragement for data collection and comparison in other Latin American countries.

Keywords

child and family welfare; comparative research; family foster care; out-of-home care

\section{Introducción}

En los últimos años ha aumentado enormemente el interés por los trabajos comparativos en el área de la investigación social, reconociéndose los múltiples beneficios de esta práctica (Baistow, 2000), ya que no solo permite aprender sobre la forma de abordar los problemas sociales de otros territorios, sino que el propio proceso de comparación puede resultar un estímulo para la creatividad y la reflexión sobre lo que puede ser mejorado en nuestro contexto.

A pesar de que en la última década se ha publicado un creciente número de investigaciones dedicadas a comparar internacionalmente los sistemas de protección infantil, estos trabajos se han centrado en países anglosajones, fundamentalmente Reino Unido, Estados Unidos y Australia (véanse Begg, 2011; Courtney \& Iwaniec, 2009; EspingAndersen et al., 2012; Gilbert, Parton \& Skivenes, 2011; Munro, Stein \& Ward, 2005; Segal, 2004; Munro \& Stein, 2008).

Sin embargo, los países del sur de Europa presentan ciertas particularidades culturales, económicas y políticas que los distancian de otros países occidentales (Castles, 1998; Ferrera, 1996). Siguiendo la popular clasificación de regímenes de bienestar capitalistas delimitada por Esping-Andersen (1990, 2000), los rasgos distintivos de estos países son el familiarismo (caracterizado por la solidaridad intergeneracional), la permanencia del modelo de varón-sustentador, un débil tejido institucional de apoyo a la familia, el envejecimiento de la población y una limitada incorporación de la mujer al mercado laboral. Estos países se caracterizan además por una polarización norte/ sur o zonas rurales/urbanas (Santos \& Ferreira, 2001), el influjo de la Iglesia católica y cierto retraso en el proceso de modernización socioeconómica, como consecuencia de la tardía transición que han realizado desde regímenes políticos autoritarios a regímenes políticos democráticos (Leibfried, 1993).

Estas peculiaridades se extienden a la práctica de la protección infantil. Una de ellas es la tardía aparición del acogimiento familiar, que en los países de la Europa central, nórdicos y anglosajones cuenta con varias décadas de implantación. Los países mediterráneos, particularmente los de fuerte influencia de la Iglesia católica, han mantenido el predominio de las instituciones de acogida de menores desprotegidos (esto explica la similaridad con países como Irlanda en esta cuestión). En España, el acogimiento familiar se introduce en el marco jurídico en 1987 y en Portugal en 1999 y todavía en la actualidad presenta cifras que indican un lento y costoso desarrollo.

Según cifras recogidas en 93 países, el número de niños que no pueden vivir con sus padres se estima en 143 millones (McCall, 2011). La mayoría de ellos (en torno al 95\%) son acogidos por los propios familiares (acogida en familia extensa) o por otras familias (acogida en familia ajena). A nivel internacional se observa una tendencia creciente en esta cifra, lo cual se ha relacionado con la crisis económica global, pero también con una mayor conciencia social sobre el bienestar de los niños y niñas y los movimientos por los derechos de la infancia (Eurochild, 2010).

El objetivo de este artículo es analizar de forma comparada el desarrollo de la medida de acogimiento familiar, en España y Portugal. Aunque ambos países representan el contexto de estudio, también serán tratados como unidad de análisis a la hora de examinar cómo el desarrollo de esta intervención se puede ver afectado por ciertas características del país en cuestión. Para esto, a 
continuación se ofrece una panorámica de ambos sistemas de protección que proporciona un conocimiento esencial para la posterior interpretación de los resultados. Posteriormente, se exponen los resultados de un estudio empírico que ha tratado de revelar los perfiles y procesos característicos del acogimiento familiar.

\section{El acogimiento familiar en España}

En poco más de dos décadas España ha evolucionado desde un modelo de beneficencia, caracterizado por políticas de institucionalización de la infancia en riesgo, a un sistema de protección infantil comparable a los países europeos más avanzados, si bien todavía se puede observar cierto retraso en el desarrollo de algunos programas, como el que aquí ocupa nuestra atención.

Dos son los desarrollos legales que dan el empuje definitivo a la introducción de la medida de acogimiento familiar en España. Inicialmente, la Ley 21/87 introduce la posibilidad de la acogida familiar en un momento en el que la protección en España se ejercía casi exclusivamente a través de la institucionalización de aquellos menores que no podían continuar viviendo con sus progenitores. Posteriormente, la Ley Orgánica 1/96 de Protección Jurídica del Menor trató de promover la medida, ofreciendo diferentes alternativas de acogida en función de las distintas necesidades de los niños y las familias, distinguiendo tres modalidades: simple, permanente y preadoptivo; a la vez que introducía la posibilidad de acordar un acogimiento provisional. A través de estas dos reformas legislativas, el acogimiento familiar se consolidaba como la opción más deseable para aquellos niños que debían ser separados de sus familias, especialmente para los más pequeños.

Sin embargo, el ingreso en una residencia como medida de protección ha sido la forma casi exclusiva de intervención hasta tiempos recientes, aunque los hogares actuales difieren enormemente de las instituciones del pasado. El acogimiento residencial, en la actualidad, responde a un modelo especializado en perfiles de cierta complejidad como adolescentes sin previsión de retorno al hogar familiar; niños con problemas emocionales, de conducta y salud men- tal; adolescentes que presentan conductas violentas hacia su familia; menores infractores con medida protectora y menores extranjeros no acompañados (Bravo \& Del Valle, 2009). Para un análisis de su evolución y características en el contexto español se recomienda consultar Del Valle y Casas (2002) y Bravo y Del Valle (2009).

El acogimiento familiar supone una medida más normalizadora, ya que el niño se integra en otro hogar con unas figuras parentales y ambiente de tipo familiar. Esta intervención cuenta con diferentes modalidades, empezando por la distinción básica entre acogimiento simple, definido como una medida de carácter transitorio que permite a un menor ser acogido en una familia mientras se soluciona la situación que provocó su salida de su núcleo familiar; y el acogimiento permanente, para las situaciones en las que no es viable la reunificación del menor dentro con su familia de origen ni es posible la adopción. A estas modalidades se sumaría la del acogimiento provisional, mediante la cual la entidad pública puede llevar a cabo un acogimiento familiar sin el consentimiento de los padres.

Respecto a la radiografía en términos estadísticos del sistema de protección español, a priori se puede afirmar que ingresan más niños en acogimiento residencial que en acogimiento familiar, revelándose además una tendencia creciente en los últimos años que se ha relacionado con la aparición de nuevos perfiles de protección (Bravo \& Del Valle, 2009). Sin embargo, cuando se toma la cifra de casos que permanecen acogidos a final de año, se observa que en 2010, 14.088 niños se encontraban en acogimiento residencial y 20.481 en acogimiento familiar (Observatorio de la Infancia, 2012), lo que nos indicaría que un $60 \%$ de los niños se encuentra acogido en familias. Otra cuestión de gran importancia tiene que ver con el tipo de familia acogedora en términos de parentesco (ajena o extensa). Este dato, que no puede consultarse en la estadística básica de medidas de protección a la infancia, arrojaba una proporción del $80 \%$ con familia extensa en el año 2002 (Del Valle \& Bravo, 2003).

La forma en que ha transcurrido la implantación y desarrollo del acogimiento en familia ajena en España cuenta con escasa investigación, por lo que 
merecen especial atención los trabajos evaluativos de Amorós, Palacios, Fuentes, León y Mesas (2003), Del Valle y Bravo (2003), Del Valle et al. (2008) y Jiménez y Palacios (2008). Otros aspectos específicos del acogimiento familiar han sido analizados en las publicaciones de Balluerka, Gorostiaga, Herce y Rivero (2002) sobre la integración del menor acogido en su familia acogedora; Fernández-Molina, Del Valle, Fuentes, Bernedo y Bravo (2011) sobre los problemas de conducta de los adolescentes en acogimiento; Salas, Fuentes, Bernedo, García y Camacho (2009) sobre las visitas de los menores con sus padres biológicos; Bernedo y Fuentes (2010) y Montserrat (2012) sobre los acogimientos con familia extensa.

Las conclusiones de la investigación española reciente sobre acogimiento familiar apuntan a que esta medida se caracteriza por la alta estabilidad y permanencia que ofrece a los menores acogidos (Del Valle, López, Montserrat \& Bravo, 2009); el reducido número de rupturas de la acogida, especialmente cuando el menor se encuentra en su familia extensa (López, Del Valle, Montserrat \& Bravo, 2010); la prolongación de las estancias, con la consiguiente disminución de posibilidades de retorno a la familia biológica (López, Montserrat, Bravo \& Del Valle, 2013); y la continuación de la convivencia con los acogedores una vez alcanzada la mayoría de edad (López, Santos, Bravo \& Del Valle, 2013).

En un reciente estudio sobre niños que esperan por un acogimiento familiar en recursos residenciales, uno de los resultados más llamativos fue la gran cantidad de dificultades comportamentales que presentan (López \& Del Valle, 2013; López et al., 2010); un 26\% de los niños en acogimiento residencial estaba recibiendo o había recibido tratamiento por trastorno de salud mental y un $17 \%$ del total presentaba discapacidad intelectual (Sainero, Bravo \& Del Valle, 2013).

\section{El acogimiento familiar en Portugal}

El sistema de protección a la infancia en Portugal experimentó una importante reforma a finales del siglo XX. Hasta ese momento, junto con las situaciones de desprotección infantil se incluía la delincuencia juvenil y otras realidades asociadas. Para todos los casos, se preveía el mismo proceso y las mismas respuestas, incluyendo el internamiento en las mismas instituciones encargadas de la protección de menores. Hasta entonces, una de las características más remarcables del sistema de protección infantil portugués era el uso casi exclusivo del acogimiento residencial, una intervención que en este contexto ha sido sinónimo de institucionalización, restringiendo significativamente los derechos del niño y de sus padres (Delgado, 2006).

Desde 1999, el sistema de protección portugués se divide en dos tipos de respuestas: las medidas para la promoción y protección integradas en la Ley de protección de los niños y jóvenes en riesgo (Ley n.o 147/99, de 1 de septiembre) y las medidas sancionadoras-educativas relacionadas con los delitos tipificados en la Ley tutelar educativa (Ley n. ${ }^{\mathrm{o}}$ 166/99, de 14 de septiembre).

A partir de esta reforma legislativa, en el ámbito de la protección portugués, se pasa a distinguir entre el concepto de "riesgo" y de "peligro" (este último equivalente a la situación de desamparo en España), bajo la premisa de que la situación de riesgo no siempre justifica por sí misma la intervención protectora (Carvalho, 2004; Guerra, 2000).

El objetivo de la intervención ha de ser en todas las situaciones asegurar el desarrollo físico, moral y psicológico del niño, según corresponda a su edad. Las medidas aplicadas pueden realizarse en el medio natural del niño (a través de intervención familiar), o pueden referirse a un acogimiento, ya sea familiar, en un hogar residencial o con miras a su adopción futura (acogimiento en familia preadoptiva). Al igual que ocurre en el sistema de protección español, las medidas de acogimiento en familia o institución se limitan a casos graves y son el último recurso, ya que la intervención solo ha de interferir con la vida del niño y su familia cuando sea estrictamente necesario.

Los principios rectores de la intervención, sus objetivos, las medidas aplicables, las entidades responsables de la intervención, entre otros elementos, han sido objeto de varios estudios (Delgado, 2006; Furtado \& Guerra, 2000; Gersão, 2000; Guerra, 
2003, 2004), así como la caracterización del proceso de protección (Delgado, 2006; Pinto, 2006).

Con la entrada en vigor del nuevo marco normativo en materia de acogimiento familiar, contenido en el Decreto-Ley n. N.. 11/2008, de fecha 17 de enero, el acogimiento en familia extensa, es decir, de personas unidas al niño por lazos familiares, deja de ser clasificado legalmente como acogimiento familiar, pasando a ser considerado una medida que tiene lugar en el medio natural de vida del niño.

Según el informe anual de caracterización de los niños y jóvenes en acogida en Portugal (Instituto da Segurança Social, 2012), en 2011 se encontraban en protección 8.938 niños. Un total de 5.834 niños se encontraba acogido en hogares de infancia y juventud, 2.144 en los centros de acogimiento temporal (creados para acogidas de corta duración) y solo 485 estaban en acogimiento con familia ajena. El resto de niños se encontraba distribuido por otras respuestas de tipo institucional especializado (acogimiento de emergencia, comunidad terapéutica, pisos de preparación para la independencia, hogares especializados en discapacidad, centros para padres adolescentes, comunidades de inserción, etc.). Además, en el año 2007, último año en que contamos con datos oficiales, había 979 niños acogidos con su familia extensa (Instituto da Segurança Social, 2008).

Respecto a la relación entre la edad de los menores y el tipo de recurso, sorprende comprobar cómo la mayoría de los niños entre 0 y 3 años son admitidos en centros de acogimiento temporal (79\%), un porcentaje menor va para hogares residenciales de diferentes tipos (20\%) y un número residual para acogimiento familiar (1\%) (Instituto da Segurança Social, 2012).

En relación con las características de la población atendida en el sistema de protección portugués, se constata que una proporción significativa exhibe problemas de conducta (18\%) y presenta fracaso escolar (el 49\% de los niños mayores de 13 años). Un número importante muestra problemas de salud mental (12\%), discapacidad intelectual (13\%) o abuso de drogas (8\%) (Instituto da Segurança Social, 2012). Se trata de situaciones complejas que requieren respuestas especializadas y personal cualificado, tanto en las instituciones de acogida como en las familias acogedoras, ya que los acogedores deben contar con las habilidades, recursos y supervisión necesarios para estimular la recuperación de estos menores.

Las cifras revelan un sistema de protección centrado en la medida de acogimiento residencial que recibe aproximadamente el 95\% de los niños, una tendencia que se ha incrementado en los últimos años y muestra un panorama sin parangón en otros países de la Unión Europea (Eurochild, 2010). Ante estas cifras resulta evidente que el cambio producido en la mayoría de los países occidentales, con la desaparición de las grandes instituciones de acogida y la apuesta por el acogimiento familiar, está aún pendiente de realización en Portugal.

Treinta años después de su introducción desde un punto de vista legal, el acogimiento familiar se caracteriza por la escasez de campañas de promoción y difusión de la medida, la existencia de deficiencias en materia de selección y apoyo técnico a los acogedores, la falta de formación inicial para las familias candidatas, el bajo nivel económico y educativo de las familias de acogida y la falta de asociaciones que representen y apoyen a estas familias (Delgado, 2007).

Frente a esta realidad, la comunidad científica portuguesa ha centrado la escasa investigación sobre protección infantil en otros aspectos de la intervención socioeducativa, como la adopción o el problema de la violencia. Son casi inexistentes los estudios que se ocupan del acogimiento familiar, por lo que se reconoce especialmente el mérito de los trabajos de Cardoso (2010), Delgado (2007, 2008, 2011), Martins (2005), Pinto (2008) y Tribuna y Relvas (2002).

\section{Método}

\section{Instrumento}

El instrumento empleado en esta comparativa fue desarrollado por el Grupo de Investigación en Familia de Infancia de la Universidad de Oviedo con motivo de la evaluación estatal de la medida de acogimiento familiar en España (se puede consultar en Del Valle, López, Montserrat \& Bravo, 2008). Fue confecciona- 
do a partir de la revisión de la investigación internacional, incluyendo preguntas cerradas y abiertas de carácter más descriptivo, y se centró en la recogida de datos sobre los perfiles de los protagonistas del acogimiento familiar (niños, familias biológicas y familias acogedoras), así como los episodios de acogida.

Los datos recogidos a través del instrumento que se presentan en este artículo hacen referencia a: (a) Niños: sexo, edad al inicio del acogimiento, edad al abrir el caso de protección, discapacidades y enfermedades, origen nacional o étnico; (b) Familias de origen: situación de pareja, problemas psicosociales en padre y madre; (c) Familias acogedoras: edad, situación de pareja, nivel educativo, hijos propios, acogimientos previos, acogimiento múltiple y (d) Episodio: situación previa, temporalidad, intervención judicial, visitas, lugar de las visitas, actitud de la madre y el padre ante la intervención protectora.

Posteriormente, el equipo de la Universidad de Porto replicó el estudio siguiendo el diseño español y aplicando la hoja de recogida de datos adaptada al contexto portugués. Las mismas variables fueron recogidas en ambos países, si bien existen ciertas especificaciones respecto a los requisitos aplicados para la selección muestral que serán expuestas a continuación.

\section{Muestra}

En España, la muestra quedó formada por 357 casos de acogimiento familiar pertenecientes a cinco de las diecisiete comunidades autónomas españolas, las cuales realizan más del 70\% de los acogimientos llevados a cabo en este país. Los casos fueron seleccionados al azar de entre aquellos que habían sido abiertos durante los 5 años previos al inicio del estudio (año 2006) y que permanecían abiertos en dicho momento, siendo proporcionales al número de acogimientos de cada comunidad autónoma. Se excluyeron de la selección los acogimientos de hecho, especializados y pre-adoptivos, por corresponder a realidades muy específicas que se alejaban del interés del estudio.

En Portugal la muestra incluyó todos los casos de acogimiento familiar que se encontraban abiertos en mayo de 2011 en el distrito de Porto (289 casos; lo que representa cerca del 52\% del total de acogimientos realizados en Portugal) y cuya supervisión estaba a cargo del Equipo de Seguridad Social de Porto. Al igual que en la muestra española, se excluyeron los casos de acogimiento familiar de hecho y pre-adoptivo; sin embargo, la muestra incluye algunos casos de acogimientos especializados (tipología que no fue incluida en España).

Teniendo en cuenta lo expuesto sobre la selección muestral en ambos países, se reconoce que esta comparativa afronta dos limitaciones metodológicas que no parecen comprometer de forma grave la valía de los resultados. En primer lugar, existe una diferencia temporal de cinco años entre la recogida de datos en ambos países (los casos españoles se recogen en el año 2006 y los de Portugal en 2011). A pesar de ello, se ha podido comprobar que en los últimos cinco años las estadísticas no revelan cambios sustanciales en el desarrollo de esta medida de protección en ninguno de los dos países. En segundo lugar, en España la muestra se recoge en diferentes territorios del Estado, de modo que la generalizabilidad de los resultados es mayor, mientras que los casos de Portugal representan un único distrito (Porto). A pesar de ello, atendiendo a la uniformidad sociológica y cultural de este país y al hecho de que se ha incluido $52 \%$ de los acogimientos familiares llevados a cabo en Portugal, se considera que la muestra es razonablemente representativa.

\section{Procedimiento}

En el estudio español, la muestra se recogió con la ayuda de cinco investigadores entrenados para tal efecto, que se desplazaron a cada región para tomar los datos mediante la lectura de los expedientes y la realización de entrevistas con los profesionales responsables de cada caso. En Portugal, los datos fueron recogidos por los propios miembros de los equipos de Seguridad Social responsables del seguimiento de los acogimientos familiares.

Las diferencias entre medidas comparadas entre ambos países en las variables seleccionadas se examinaron utilizando pruebas para valorar la significación estadística de las diferencias entre valores absolutos (prueba t para igualdad de medias) y de la 
diferencia entre proporciones (prueba $z$ ), así como para verificar la independencia entre algunas de las variables para los dos países (prueba de chi cuadrado). Para todas las pruebas estadísticas, se consideró el valor máximo del 5\% para el error de tipo I. Los análisis se realizaron con SPSS 20.0.

\section{Resultados}

\section{Perfil de los niños acogidos}

La distribución por sexos en ambos países resultó muy similar, con un número muy parecido de niños y niñas (Tabla 1). Sin embargo, importantes diferencias emergieron al analizar la variable edad del niño al inicio de la acogida, ya que esta fue significativamente más baja en Portugal con una media de $5.62(\mathrm{DE}=4.55)$, frente a los $6.95(\mathrm{DE}$ $=4.56)$ años de los niños acogidos en España. Esta tendencia se observó igualmente al revisar la distribución de casos por periodos evolutivos, ya que un porcentaje ligeramente superior de niños se situó en el rango de edad más bajo en el caso de Portugal (edad maternal, de 0 a 3 años), aunque las diferencias no llegaron a ser estadísticamente significativas.

Se hallaron diferencias significativas respecto a la edad en el momento de la apertura del caso, que fue 9 meses superior en Portugal $(M=5.32$; DE = 4.56). Del mismo modo, la distribución dentro de los periodos evolutivos mostró una asociación estadísticamente significativa con el país de procedencia, siendo superior en Portugal el porcentaje de niños que en el momento de apertura del caso se encontraban en los grupos de edades más elevadas.

Respecto a las problemáticas presentes en los niños acogidos, se observó que el porcentaje de casos con discapacidad en Portugal era el doble que en España, aunque ocurrió lo contrario al analizar la presencia de casos con enfermedades graves, cuya aparición fue superior en la muestra española. Es conveniente recordar aquí que la selección muestral en Portugal incluyó casos definidos como especializados, que precisamente fueron excluidos de la muestra española; esto explicaría la mayor presencia de discapacidad entre los niños portugueses.

En España, aunque un número superior de niños acogidos mostró una procedencia nacional diferente de la española, esta diferencia no llegó

\section{TABLA 1}

Variables del perfil de los niños acogidos

\begin{tabular}{lccc}
\hline & & España & Portugal \\
\hline Sexo (\%) & Niñas & 49.9 & 48.8 \\
\hline Edad inicio acogida* & & 6.95 & 5.62 \\
Distribución grupos edad* (\%) & $0-3$ & 31.2 & 40.1 \\
& $4-8$ & 35.4 & 32.9 \\
& $9-12$ & 22.4 & 16.6 \\
& +12 & 11 & 10.4 \\
Edad apertura del caso & & 4.57 & 5.32 \\
Distribución grupos edad* (\%) & $0-3$ & 51.4 & 42.9 \\
& $4-8$ & 33.1 & 29.8 \\
& $9-12$ & 12.0 & 15.6 \\
Discapacidades**(\%) & +12 & 3.4 & 11.8 \\
Enfermedades graves*** (\%) & & 8.1 & 17.3 \\
Otra nacionalidad (\%) & & 9.2 & 4.8 \\
\hline \% & & 6.8 & 4.2 \\
\hline
\end{tabular}

$* p<0.001\left(t / \chi^{2}\right) . * * p<0.01\left(t / \chi^{2}\right) . * * * p<0.05\left(t / \chi^{2}\right)$.

Fuente: elaboración propia 
a ser estadísticamente significativa respecto a la muestra portuguesa.

\section{Perfil de las familias de origen}

El estudio de las características de las familias de origen se realizó sobre la muestra de familias y no de niños, para evitar la sobrerrepresentación de los casos de padres de grupos de hermanos acogidos. Los datos de este apartado describen el perfil de 344 familias españolas y 214 portuguesas (una vez extraídos los casos en los que no se contaba con información sobre los padres o estos habían fallecido).

En ambas muestras destacó el alto grado de rupturas familiares (Tabla 2), alcanzando a la mitad de la muestra en el caso de Portugal. La situación de madre soltera fue casi dos veces superior en el caso español. El alcoholismo, tanto del padre como de la madre, se reveló como una de las problemáticas más frecuentes en las familias de origen portuguesas (69\% en los padres y $28 \%$ en las madres), mientras que entre las españolas la adicción a otras sustancias resultó más habitual (18\% en los padres y $26 \%$ en la madres).
Las situaciones familiares de prisión parecen condicionar la entrada en acogimiento familiar en mayor medida para los casos que componen la muestra española, presentándose esta problemática con una presencia significativamente superior que en la muestra portuguesa. Una dificultad que emergió con una alta frecuencia en ambas muestras fue la existencia de problemas mentales en la madre (20\% en España y 28\% en Portugal).

\section{Perfil de las familias acogedoras}

Para el análisis de los perfiles de las familias acogedoras (Tabla 3) se trabajó con una muestra reducida respecto a la de niños, al coincidir algunos de ellos acogidos en la misma familia. La muestra es en este caso de 343 casos en España y 168 en Portugal. Los acogedores portugueses presentaron una edad significativamente superior a la de los españoles, siendo la diferencia en torno a 8 años superior tanto en el caso de los hombres como de las mujeres.

La distribución de la tipología de familia acogedora resultó muy similar en ambos países, siendo la

TABLA 2

Variables del perfil de las familias de origen

\begin{tabular}{llcc}
\hline & & $\begin{array}{c}\text { Espana } \\
(\%)\end{array}$ & $\begin{array}{c}\text { Portugal } \\
(\%)\end{array}$ \\
\hline Estado pareja & Casada & 28.2 & 21.2 \\
& Divorciada & 38.8 & 50.6 \\
& Viudedad & 10.7 & 15.4 \\
Padre & Madre soltera & 22 & 12.8 \\
& Abuso de drogas*** & 18 & 9.1 \\
& Alcoholismo* & 11.6 & 68.8 \\
& Prisión*** & 14 & 6.5 \\
Madre & Pobreza & 13.7 & 13 \\
& Salud mental & 3.8 & 3.9 \\
& Enfermedad crónica & 2 & 5.2 \\
& Abuso de drogas* & 25.6 & 7.7 \\
& Alcoholismo* & 9.6 & 27.9 \\
& Prisión** & 14.1 & 2.9 \\
& Pobreza & 27.6 & 20.2 \\
& Salud mental & 20.1 & 27.9 \\
& Prostitución & 9.6 & 15.4 \\
& Enfermedad crónica & 6.4 & 3.8 \\
\hline
\end{tabular}

$* p<0.001(z) . \quad * * p<0.01(z) . * * * p<0.05(z)$.

Fuente: elaboración propia 
situación más habitual acoger en pareja (en torno al 80\% de los casos). Sin embargo, importantes diferencias emergieron al analizar el nivel educativo de las familias acogedoras, ya que este fue superior en el caso de los acogedores españoles. La gran mayoría de los acogedores portugueses mostró un nivel de estudios básico, mientras que en el caso español tres cuartas partes habían alcanzado un nivel educativo medio o alto.

En Portugal, un número significativamente superior de familias había acogido tras tener hijos, siendo habitualmente mayores que los niños acogidos. Además, las situaciones en las que se habían producido acogidas anteriores al caso estudiado o en las que existía un acogimiento múltiple (varios niños acogidos simultáneamente) fueron significativamente más frecuentes en Portugal.

\section{El episodio de acogimiento}

La proporción de niños que había tenido una estancia en un hogar residencial antes de la acogida familiar fue significativamente superior en la muestra española. La medida previa más frecuente en el caso de Portugal fue la intervención familiar, de apoyo psicopedagógico o social sobre los padres u otro familiar, para el $41 \%$ de los casos.

Se observaron diferencias estadísticamente significativas al analizar los casos en los que se requirió una intervención judicial para la puesta en marcha del acogimiento, siendo la proporción superior en el caso de Portugal (en torno al 80\% de los casos frente al 55\% en España).

Al analizar el contacto familiar, se observó que una proporción significativamente superior de niños mantenía visitas con sus progenitores en el caso de Portugal. Los escenarios del contacto familiar también fueron diferentes en función del país, siendo habitual para España que se realizaran en los llamados puntos de encuentro (39\%) o en el hogar de la familia de origen (33\%); mientras que en Portugal fueron más frecuentes las visitas en el hogar de los acogedores (56\%) o el hogar de la propia familia de origen del menor (45\%).

Respecto a la actitud de los padres sobre la intervención, en la muestra portuguesa ambos progenitores se mostraron en mayor medida ausentes. La oposición destacó especialmente entre ambos padres en el caso de España.

TABLA 3

Variables del perfil de las familias acogedoras

\begin{tabular}{lccc}
\hline & & España & Portugal \\
\hline Edad* & Acogedor & 47.7 & 55.9 \\
Tipo de familia & Acogedora & 46.2 & 53.7 \\
& & $(\%)$ & $(\%)$ \\
& Pareja & 82.9 & 82.4 \\
& Mujer sola & 14.1 & 17.3 \\
Nivel educativo* & Hombre solo & 3 & 0.3 \\
& Alto & 40.3 & 2 \\
& Medio & 32.2 & 4.7 \\
& Primario & 26 & 91 \\
Con hijos* & Sin estudios & 0.6 & 2.3 \\
Hijos mayores que los acogidos* & & 59 & 92.9 \\
Acogida previas* & & 77 & 92.9 \\
Acogida múltiple* & & 9 & 44 \\
\hline
\end{tabular}

$* p<0.001\left(t / \chi^{2}\right)$.

Fuente: elaboración propia 
TABLA 4

Variables del episodio de acogimiento

\begin{tabular}{llcc}
\hline & & $\begin{array}{c}\text { España } \\
(\%)\end{array}$ & $\begin{array}{c}\text { Portugal } \\
(\%)\end{array}$ \\
\hline Situación previa & Acogimiento residencial* & 69.5 & 5.5 \\
& Intervención familiar & 18.2 & 40.8 \\
Previsión temporal & Larga estancia* & 54.9 & 64.9 \\
Intervención judicial* & & 46.5 & 80.6 \\
Contacto familiar** & & 64.1 & 57.8 \\
Lugar de las visitas & Hogar acogedores*** & 6.8 & 56.3 \\
& Punto de encuentro & 38.5 & 0 \\
& Hogar familia origen*** & 18.2 & 45 \\
& Lugar público** & 32.8 & 9.4 \\
Actitud de la madre* & Otro & 15.6 & 1.9 \\
& Ausente & 29.4 & 50.3 \\
& Cooperación & 34.6 & 31.3 \\
& Oposición & 17.8 & 9.2 \\
Actitud del padre* & Ambigüedad & 18.2 & 9.2 \\
& Ausente & 54.7 & 68.5 \\
& Cooperación & 18.4 & 18 \\
& Oposición & 17.5 & 5.6 \\
& Ambigüedad & 9.4 & 7.9 \\
\hline
\end{tabular}

$* p<0.001\left(t / \chi^{2}\right) . * * p<0.01\left(t / \chi^{2}\right) . * * * p<0.05\left(t / \chi^{2}\right)$.

Fuente: elaboración propia

\section{Discusión y conclusiones}

La investigación comparativa aquí presentada evidencia la existencia de diferencias en los perfiles y procesos de acogida familiar en España y Portugal. Respecto a los protagonistas de la medida, se ha constatado el comienzo de la protección en torno a los cuatro años y medio en España y casi cinco y medio en Portugal. A pesar de ello, la entrada en acogimiento se produce a una edad más baja en el caso de los niños portugueses (situándose cerca a los siete años en España y poco más de cinco y medio en Portugal). Por lo tanto, el tiempo transcurrido desde la apertura del caso hasta que el niño es acogido no llega a los cuatro meses en Portugal, pero asciende hasta casi dos años y medio en España. Esta diferencia podría deberse a que los niños en Portugal pasan directamente a ser acogidos en familia sin necesidad de ser alojados, en una fase inicial, en un hogar residencial. En cambio, en España parece requerirse el paso por un hogar residencial en un gran número de casos, posiblemente por la lentitud de trámites y la dilación de los procesos de toma de decisiones (ya que legalmente sí es posible pasar directamente a un acogimiento familiar). La rapidez en el procedimiento portugués se valoraría muy positivamente, ya que diversos estudios demuestran como a medida que la edad del acogido es más alta, el acogimiento presenta mayor probabilidad de ruptura (Harwin, Owen, Locke \& Forrester, 2003; James, Landsverk \& Slymen, 2004; López et al., 2011; Webster, Barth \& Needell, 2000). Sería necesario analizar en profundidad los procesos de toma de decisiones en Portugal para determinar si los protocolos de intervención de este país pueden ofrecer pautas de mejora para el caso español.

El estudio de perfiles muestra un importante número de niños con discapacidades (especialmente en Portugal, donde no se han excluido de la muestra casos de especial necesidad), en congruencia con diversas investigaciones que han constatado como los niños con una medida de protección infantil padecen mayores déficits en su estado de salud física y mental que la población general pediátrica y ado- 
lescente (Chernoff, Combs-Orme, Risely-Curtiss \& Heisler, 1994; Dubner \& Motta, 1999; Dubowitz, Feigelman, Harrington \& Starr, 1994; Schneider \& Phares, 2005).

La presencia de estas dificultades entre los niños acogidos, que se han asociado a un mayor riesgo de ruptura del acogimiento (Leslie et al., 2005; Parker, 1966) y a una menor probabilidad de reunificación familiar (Courtney, 1994; Teare, Becker-Wilson \& Larzelere, 2001; Wade, Biehal, Farrelly \& Sinclair, 2010), sugiere la necesidad de dirigir los esfuerzos de captación hacia perfiles muy concretos de familias acogedoras, con ciertas habilidades educativas que puedan ser reforzadas a través de esquemas más intensos de preparación y apoyo técnico. Sin embargo, al analizar el perfil de los acogedores, especialmente en el caso de Portugal, no parece tratarse de un grupo con suficientes recursos para hacerse cargo de niños con necesidades especiales. Los acogedores portugueses conforman un grupo de edad avanzada, con un bajo nivel educativo y que llevan a cabo la acogida de más de un niño simultáneamente. En cambio, las familias españolas parecen afrontar la acogida en una situación más ventajosa: con un mayor nivel educativo, una edad en torno a ocho años inferior a los portugueses y situaciones de acogida de un solo niño en la mayoría de los casos, todo ello coincidente con otros estudios españoles (Amorós et al., 2003; Del Valle et al., 2008; Jiménez \& Palacios, 2008; Molero, Moral, Albiñana, Sabater \& Sospedra, 2007; Montserrat, 2006). En ambos países las familias acogedoras responden a una estructura familiar tradicional de pareja con hijos, como ocurre en otros estudios internacionales (Triseliotis, Borland \& Hill, 2000).

El perfil de acogedores retratado en Portugal sugiere que pronto será necesario llevar a cabo una renovación generacional del banco de acogedores a través de la selección de nuevas familias con un nivel educativo y unas habilidades que les permitan responder de manera más adecuada a los retos propios del acogimiento familiar. La gestión de los contactos con las familias de origen y la terminación de la acogida son dos ejemplos de los desafíos propios de una intervención que requiere no solo de la generosidad y entrega de estas familias, sino también de una clara definición del papel de acogedor, de su formación continua y del trabajo en red con los profesionales de los equipos de acogida (Triseliotis, Sellick \& Short, 1995).

Un dato de especial relevancia respecto al análisis del perfil de las familias acogedoras ha sido su experiencia previa de acogida y la existencia de acogimientos múltiples, que han resultado ser mucho más frecuentes en el contexto luso. Podría decirse que los acogedores portugueses atesoran una experiencia acumulada a lo largo de varios años de colaboración con los programas, tratándose en muchas ocasiones de acogidas de varios niños. Sería interesante conocer si era este el objetivo que perseguían inicialmente los acogedores o si han acabado asumiendo esta tarea por imposición de los servicios de acogida. Otra cuestión igualmente relevante es si la permanencia en el programa de acogidas se debe a una alta satisfacción con este y sus profesionales. Por tanto, cuestiones como su nivel satisfacción, los factores que les mantienen comprometidos con el programa o los sentimientos de sobrecarga o estrés generados por la acogida deberán ser objeto de atención de fututos trabajos.

Es destacable que en el caso español solo un 9\% de las familias acogedoras ha tenido un episodio previo de acogida de otro menor, lo cual se explica por la alta permanencia del niño en acogida y su incorporación a la familia como un miembro más. El acogimiento permanente o a largo plazo es la intervención más habitual en los dos países estudiados, dejando al descubierto programas de acogida que se basan en la permanencia y la estabilidad, al contrario de lo que ocurre en otros países donde los niños acogidos sufren numerosos cambios y transiciones (Bullock, Courtney, Parker, Sinclair \& Thoburn, 2006; Delgado, 2006; Sinclair, Baker, Lee \& Gibbs, 2007; Ward, Skuse \& Munro, 2005). Se sabe que en ambos países son muy escasas las situaciones en las que el menor regresa con su familia de origen o en que se produce un cambio de emplazamiento debido a la ruptura de la acogida familiar (Jiménez \& Palacios, 2008; Delgado, 2007, 2011; Salas et al., 2009; Tribuna \& Relvas, 2002). Parece evidente que una vez acogido, el niño se convierte en parte de la familia y permanece allí hasta la mayoría de edad 
o la independencia, lo que reduce el número de familias disponibles para acoger a otros menores (Del Valle et al., 2008; Sinclair, Gibbs \& Wilson, 2004).

Aunque en ambos países el acogimiento familiar se define como una medida de carácter transitorio, asentada en la idea prioritaria del regreso del niño a su familia de origen (en Portugal ni siquiera está previsto el acogimiento familiar de larga duración o permanente), los datos aportados por los dos países demuestran que en un número importante de casos no existe contacto del niño con la familia de origen, lo que indicaría una baja probabilidad de reunificación familiar.

Este estudio ha revelado patrones y procesos de contacto muy dispares entre los países analizados. Las visitas se llevan a cabo en mayor medida en puntos de encuentro y espacios públicos en el caso de España y en hogares, de los acogedores o las familias de origen, en el caso de Portugal. Los resultados españoles se desvían de los obtenidos en otros estudios internacionales que sitúan las visitas en el hogar de acogida entre un 20\% y $30 \%$ de las ocasiones (Cleaver, 2000; Triseliotis et al., 2000). En el caso de Portugal, será interesante explorar la utilización de otros entornos para el desarrollo de las visitas. Ya se trate de centros comunitarios o de espacios concebidos específicamente para el contacto, a semejanza de los empleados en España, estos pueden proporcionar un ambiente de proximidad controlado y fácil de supervisar, próximo geográficamente a ambas familias y cómodo para la interacción durante las visitas. Más aún, este espacio puede convertirse en un contexto idóneo para el trabajo con las familias de origen en la adquisición o mejora de determinadas habilidades parentales.

Las familias de origen revelan distintos perfiles en ambos países. Las problemáticas más habituales en la muestra española son el abuso de drogas y las situaciones de prisión, tanto en el caso del padre como la madre. Por otro lado, el alcoholismo está mucho más presente entre las familias lusas, especialmente entre los padres. Este es un dato relevante a la hora de analizar las trayectorias y resultados de la intervención, ya que la literatura científica ha identificado el abuso de sustancias por parte de los padres como uno de los factores claves que afectaría negativamente la posibilidad de que se produzca una reunificación familiar (Brook \& McDonald, 2007; Fein, 1993; Harris, 1999; Maluccio \& Ainsworth, 2003; Mapp \& Steinberg, 2007; Smith, 2003; Terling, 1999; Wade et al., 2010).

A pesar de las diferencias observadas, encontramos similitudes en el retrato de grupo de las familias de origen en los dos países, presentando los padres conductas marginales o de riesgo, problemas de salud y de baja condición social, lo que evidencia la necesidad de un intenso trabajo de prevención y acompañamiento para la recuperación de las habilidades parentales, sin las cuales la reunificación familiar no será posible.

También se hallan importantes diferencias respecto a la actitud de los progenitores hacia la intervención. Mientras las madres portuguesas se muestran fundamentalmente ausentes, las españolas cooperan o se oponen al acogimiento. $\mathrm{Cu}$ riosamente, al analizar la figura paterna, los padres de ambas muestras revelan una actitud ausente en una proporción muy semejante, en consonancia con estudios internacionales (O'Donnell, 1999), con mayor cooperación en el caso de los padres portugueses y mayor oposición entre los padres españoles.

Se puede concluir que los sistemas de protección a la infancia objeto de esta comparación presentan diferentes patrones de evolución durante las últimas tres décadas. En España, a lo largo de los años 80 y 90 se lleva a cabo una reforma legal que prioriza el acogimiento familiar como medida de protección para aquellos menores que han de ser separados de su familia de origen (Del Valle et al., 2009). Este cambio, ha contribuido al impulso de los programas de acogida, si bien su desarrollo todavía se presenta en una expresión más baja que la mayoría de los países europeos (Colton \& Williams, 2006; Eurochild, 2010).

En el caso portugués, a pesar de que la reforma legal realizada en el año 1999, que reconoce la importancia de la familia como el contexto más deseable para el desarrollo del niño, no se establecen prioridades en la elección entre los dos tipos de acogida (residencial y familiar). De este modo, el cambio del marco normativo no produce un impacto real en el número creciente de niños en ins- 
tituciones, revelando una situación que se aleja de la realidad de España y del resto de países europeos (Instituto da Segurança Social, 2012).

Parece evidente que sin el necesario esfuerzo de modernización de las instituciones y el compromiso con la profesionalización de los equipos técnicos y educativos, el cambio del paradigma de protección infantil seguirá pendiente de realización. Podría decirse que es en estos momentos cuando en ambos países comienzan a sentirse los primeros indicios de cambio. El acogimiento familiar ha comenzado a recibir cierta atención por parte de los medios de comunicación y el discurso político actual ha comenzado a hacer alusión a la necesidad de reducir el número de niños acogidos en instituciones, en sintonía con una creciente sensibilidad social sobre los problemas de la infancia y juventud y sobre la urgente necesidad de apostar por intervenciones efectivas en casos de maltrato infantil.

\section{Referencias}

Amorós, P., Palacios, J., Fuentes, N., León, E. \& Mesas, A. (2003). Familias Canguro. Una experiencia de protección a la infancia. Barcelona: Fundació La Caixa.

Baistow, K. (2000). Cross-national research: What can we learn from inter-country comparisons? Social Work in Europe, 7(3), 8-13.

Balluerka, N., Gorostiaga, A., Herce, C. \& Rivero, A. M. (2002). Elaboración de un inventario para medir el nivel de integración del menor acogido en su familia acogedora. Psicothema, 14(3), 564-571.

Begg, I. S. (2011). Comparing fostering in Scotland and Norway with an emphasis on the use of private actors. Child Care in Practice, 17(2), 131-142.

Bernedo, I. M. \& Fuentes, M. J. (2010). Necesidades de apoyo y satisfacción en los acogimientos con familia extensa. Anales de Psicología, 26(1), 95-103.

Bravo, A. \& Del Valle, J. F. (2009). Crisis y revisión del acogimiento residencial. Su papel en la protección infantil. Papeles del Psicólogo, 30(1), 42-52.

Brook, J. \& McDonald, T. P. (2007). Evaluating the effects of comprehensive substance abuse intervention on successful reunification. Research on Social Work Practice, 17(6), 664-673.
Bullock, R., Courtney, M., Parker, R., Sinclair, I. \& Thoburn, J. (2006). Can the corporate state parent? Children and Youth Services Review, 28(11), 1344-1358.

Cardoso, L. (2010). Contextos de colocação para crianças em perigo dos 0 aos 3 anos de idade: Elementos para uma análise. Tesis de Máster, Departamento de Psicología Social y de las Organizaciones del Instituto Universitario de Lisboa, Portugal.

Carvalho, M. (2004). Pelas margens, outras infâncias - crianças marginalizadas e violência. Revista Infância e Juventude, 4, 51-144.

Castles, F. (1998). Comparative public policy. Patterns of post-war transformation. Cheltenham: Edward Elgar.

Chernoff, R. G., Combs-Orme, T., Risley-Curtiss, C. \& Heisler, A. H. (1994). Assessing the health status of children entering foster care. Pediatrics, 93(4), 594-601.

Cleaver, H. (2000). Fostering family contact. London: The Stationery Office.

Colton, M. \& Williams, M. (Eds.). (2006). Global perspectives in foster family care. Lyme Regis, UK: Russell House.

Courtney, M. E. (1994). Factors associated with the reunification of foster children with their families. Social Service Review, 68, 81-109.

Courtney, M. E. \& Iwaniec, D. (Eds.). (2009). Residential care of children: Comparative perspectives. Oxford: Oxford University Press.

Del Valle, J. F. \& Bravo, A. (2003). La situación del acogimiento familiar en España. Madrid: Ministerio de Trabajo y Asuntos Sociales. Recuperado de http://www.academia.edu/911437/Del_ Valle_J.F._y_Bravo_A._2003_._La_situacion_ del_acogimiento_familiar_en_Espana._madrid_ Ministerio_de_Trabajo_y_Asuntos_Sociales

Del Valle, J. F. \& Casas, F. (2002). Child residential care in the Spanish social protection system. International Journal of Child and Family Welfare, 5(3), 112-128.

Del Valle, J. F., López, M., Montserrat, C. \& Bravo, A. (2008). El acogimiento familiar en España. Una evaluación de resultados. Madrid: Ministerio de Trabajo y Asuntos Sociales. Recuperado de http:// 
www.observatoriodelainfancia.msssi.gob.es/documentos/AcogimientofamiliaEspana.pdf

Del Valle, J. F., López, M., Montserrat, C. \& Bravo, A. (2009). Twenty years of foster care in Spain: Profiles, patterns and outcomes. Children and Youth Services Review, 31(8), 847-853.

Delgado, P. (2006). Os direitos da criança. Da participação à responsabilidad. Porto: Profedições.

Delgado, P. (2007). Acolhimento familiar. Conceitos, práticas e (in)definições. Porto: Profedições.

Delgado, P. (2008). Crianças e acolhedores. Histórias de vida em famílias. Porto: Profedições.

Delgado, P. (2011) Acolhimento familiar de crianças. Uma perspectiva ecológica. Porto: Profedições.

Dubner, A. E. \& Motta, R. W. (1999). Sexually and physically abused foster care children and posttraumatic stress disorder. Journal of Consulting and Clinical Psychology, 67(3), 367-373.

Dubowitz, H., Feigelman, S., Harrington, D. \& Starr, R. H. (1994). Children in kinship care: How do they fare? Children and Youth Services Review, 16(1-2), 85-106.

Esping-Andersen, G. (1990). The three worlds of welfare capitalism. Cambridge: Polity.

Esping-Andersen, G. (2000). Fundamentos sociales de las economías postindustriales. Barcelona: Ariel.

Esping-Andersen, G., Garfinkel, I., Han, W. J., Magnuson, K., Wagner, S. \& Waldfogel, J. (2012). Child care and school performance in Denmark and the United States. Children and Youth Services Review, 34(3), 576-589.

Eurochild. (2010). Children in alternative care. National Surveys (2.a ed.). Cork City, Ireland: Autor.

Fein, E. (1993). Last best chance: Findings from a reunification services program. Child Welfare, 72(1), 25-40.

Fernández-Molina, M., Del Valle, J. F., Fuentes, M. J., Bernedo, I. \& Bravo, A. (2011). Problemas de conducta de los adolescentes en acogimiento preadoptivo, residencial y con familia extensa. Psicothema, 23(1), 1-6.

Ferrera, M. (1996). The 'Southern Model' of welfare in social Europe. Journal of European Social Policy, 6(1), 17-37.
Furtado, L. \& Guerra, P. (2000). O novo direito das crianças e jovens. Um recomeço. Lisboa: Centro de Estudos Judiciários.

Gersão, E. (2000). As novas leis de protecção de crianças e jovens em perigo e de tutela educativa - Uma reforma adequada aos dias de hoje. Revista Infância e Juventude, 2, 9-48.

Gilbert, N., Parton, N. \& Skivenes, M. (Eds.). (2011). Child protection systems. International trends and orientations. Oxford: Oxford University Press.

Guerra, P. (2000). Os menores e os tribunais: sinfonia em dois andamentos. Revista Infância e Juventude, 3, 9-26.

Guerra, P. (2003). O novo direito das crianças e jovens - um verdadeiro recomeço. Revista Infância e Juventude, 1, 53-80.

Guerra, P. (2004). A mediação familiar e o tribunal. Revista Infância e Juventude, 4, 35-50.

Harris, M. S. (1999). Comparing mothers of children in kinship foster care: Reunification vs. remaining in care. En J. Gleeson \& C. F. Hairston (Eds.), Kinship care: Improving practice through research (pp. 145-166). Washington, DC: Child Welfare League of America.

Harwin, J., Owen, M., Locke, R. \& Forrester, D. (2003). Making care orders work: A study of care plans and their implementation. Londres: The Stationery Office.

Instituto da Segurança Social. (2012). Relatório da caracterização anual das crianças e jovens em situação de acolhimento em 2011. Lisboa: Autor.

Instituto da Segurança Social. (2008). Plano de intervenção imediata - Relatório de caracterização das crianças e jovens em situação de acolhimento em 2007. Lisboa: Autor.

James, S., Landsverk, J. \& Slymen, D. J. (2004). Placement movement in out-of-home care: Patterns and predictors. Children and Youth Services Review, 26(2), 185-206.

Jiménez, J. \& Palacios, J. (2008). Acogimiento familiar en Andalucía. Procesos familiares, perfiles personales. Granada: Consejería para la Igualdad y Bienestar Social de la Junta de Andalucía/Universidad de Sevilla. 
Leibfried, S. (1993). 'Towards a European welfare state?' En C. Jones (Ed.), New perspectives on the welfare state in Europe (pp. 133-156). Londres: Routledge.

Leslie, L. K., Gordon, J. N., Meneken, L., Premji, K., Michelmore, K. L. \& Ganger, W. (2005). The physical, developmental, and mental health needs of young children in child welfare by initial placement type. Journal of Developmental and Behavioral Pediatrics, 26(3), 177-185.

López, M. \& Del Valle, J. F. (2013, 7 de octubre). The waiting children: Pathways (and future) of children in long term residential care. British Journal of Social Work, bct130. http://dx.doi.org/10.1093/ bjsw/bct130

López, M., Del Valle, J. F., Montserrat, C. \& Bravo, A. (2010). Niños que esperan. Estudio sobre casos de larga estancia en acogimiento residencial. Madrid: Ministerio de Sanidad y Política Social. Recuperado de http://www.observatoriodelainfancia. msssi.gob.es/productos/pdf/ninosQueEsperan.pdf

López, M., Del Valle, J. F., Montserrat, C. \& Bravo, A. (2011). Factors affecting foster care breakdown in Spain. Spanish Journal of Psychology, 14(1), 111-122.

López, M., Montserrat, C., Bravo, A. \& Del Valle, J. F. (2013). Factors associated with family reunification for children in foster care. Child and Family Social Work, 18(2), 226-236.

López, M., Santos, I., Bravo, A. \& Del Valle, J. F. (2013). El proceso de transición a la vida adulta de jóvenes acogidos en el sistema de protección. Anales de Psicología, 29(1), 187-196.

Maluccio, A. N. \& Ainsworth, F. (2003). Drug use by parents: A challenge for family reunification practice. Children and Services Review, 25(7), 511-533.

Mapp, S. C. \& Steinberg, C. (2007). Birth families as permanency resources for children in long-term foster care. Child Welfare, 86(1), 29-51.

Martins, P. (2005). O acolhimento familiar como resposta de protecção à criança sem suporte familiar adequado. Revista Infância e Juventude, 4, 63-84.

McCall, R. B. (2011). Research, practice, and policy perspectives on issues of children without permanent parental care. En R. B. McCall, M. H. van IJzendoorn, F. Juffer, C. J. Groark \& V. K. Groza (Eds.), Children without permanent parents: Research, practice, and policy (pp. 223-272). Mono- graphs of the Society for Research in Child Development, Serial No. 301(76).

Molero, R. J., Moral, M. J., Albiñana, P., Sabater, Y. \& Sospedra, R. (2007). Situación de los acogimientos en familia extensa en la ciudad de Valencia. Anales de Psicología, 23(2), 193-200.

Montserrat, C. (2006). Acogimiento en familia extensa: un estudio desde la perspectiva de los acogedores, de los niños y niñas acogidos y de los profesionales que intervienen. Intervención Psicosocial, 15(2), 203-221.

Montserrat, C. (2012, 25 de octubre). Kinship care in Spain: Messages from research. Child $\mathcal{E}$ Family Social Work. http://dx.doi.org/10.1111/cfs.12028

Munro, E. R. \& Stein, M. (2008). Comparative exploration of care leavers' transition to adulthood: An introduction. En M. Stein \& E. R. Munro (Eds.), Young people's transitions from care to adulthood: International research and practice (pp. 11-21). London: Jessica Kingsley Publishers.

Munro, E. R., Stein, M. \& Ward, H. (2005). Comparing how different social, political and legal frameworks support or inhibit transitions from public care to independence in Europe, Israel, Canada and the United States. International Journal of Child Welfare, 8(4), 191-202.

O'Donnell, J. M. (1999). Involvement of African American fathers in kinship foster care services. Social Work, 44(5), 428-441.

Observatorio de la Infancia. (2012). Boletín de datos estadísticos de medidas de protección a la infancia (№ 13). Madrid: Dirección General de Servicios para la Familia y la Infancia.

Parker, R. (1966). Decision in child care: A study of prediction in fostering. London: Allen \& Unwin.

Pinto, A. (2006). Guia de procedimentos do processo de promoção e protecção. Coimbra: Almedina.

Pinto, V. (2008). Critérios para seleção e formação de famílias de acolhimento. Tesis de Máster, Instituto Superior de Psicología Aplicada de Lisboa, Portugal.

Sainero, A., Bravo, A. \& Del Valle, J.F. (2013, 11 de enero). Examining needs and referrals to mental health services for children in residential care in Spain: An empirical study in an autonomous community. Journal of Emotional and Behavioral Disorders. http://dx.doi.org/10.1177/1063426612470517 
Salas, M. D., Fuentes, M. J., Bernedo, I. M., García, M. A. \& Camacho, S. (2009). Acogimiento en familia ajena y visitas de los menores con sus padres biológicos. Escritos de Psicología, 2(2), 35-42.

Santos, B. \& Ferreira, S. (2001). A reforma do EstadoProvidência entre globalizações conflituantes. En P. Hespanha \& G. Carapinheiro (Eds.), Risco Social e incerteza. Pode o Estado Social recuar mais? (pp. 177-226). Santa Maria da Feira: Afrontamento.

Schneider, K. M. \& Phares, V. (2005). Coping with parental loss because of termination of parental rights. Child Welfare, 84(6), 819-842.

Segal, U. A. (2004). Child Welfare programs and services: A comparison of the USA and Japan. International Social Work, 47(3), 370-390.

Sinclair, I., Gibbs, I. \& Wilson, K. (2004). Foster carers. Why they stay and why they leave. Londres: Jessica Kingsley Publishers.

Sinclair, I., Baker, C., Lee, J. \& Gibbs, I. (2007). The pursuit of permanence: A study of the English care system. Londres: Jessica Kingsley Publishers.

Smith, B. (2003). After parental rights are terminated: Factors associated with exiting foster care. Children and Youth Services Review, 25(12), 965-985.

Teare, J. F., Becker-Wilson, C. Y. \& Larzelere, R. E. (2001). Identifying risk factors for disrupted family reunifications following short-term shelter care.
Journal of Emotional and Behavioral Disorders, 9(2), 116-122.

Terling, T. (1999). The efficacy of family reunification practices: Reentry rates and correlates of reentry for abused and neglected children reunited with their families. Child Abuse $\mathbb{E}$ Neglect, 23(12), 13591370.

Tribuna, F. \& Relvas, A. (2002). Famílias de acolhimento e vinculação na adolescência. En A. Relvas \& M. Alarcão (Eds.), Novas formas de família (pp. 53-120). Coimbra: Quarteto.

Triseliotis, J., Borland, M. \& Hill, M. (2000). Delivering foster care. Londres: BAAF.

Triseliotis, J., Sellick, C. \& Short, R. (1995). Foster care: Theory and practice. Londres: Batsford.

Wade, J., Biehal, N., Farrelly, N. \& Sinclair, I. (2010). Maltreated children in the looked after system: A comparison of outcomes for those who go home and those who do not (Research brief). Londres: Department for Education.

Ward, H., Skuse, T. \& Munro, E. R. (2005). The best of times, the worst of times: Young people views of care and accommodation. Adoption and Fostering, 29(1), 8-17.

Webster, D., Barth, R. P. \& Needell, B. (2000). Placement stability for children in out-of-home care: A longitudinal analysis. Child Welfare, 79(5), 614-632. 